\title{
Civilisations
}

Revue internationale d'anthropologie et de sciences

humaines

$60-2 \mid 2012$

Être ou ne pas être balkanique

\section{L' Homo Balkanicus en contexte migratoire}

Un modèle d'identification à défendre ou à rejeter ?

\section{Katerina Seraidari}

\section{(2) OpenEdition}

Journals

Édition électronique

URL : http://journals.openedition.org/civilisations/3027

DOI : 10.4000/civilisations.3027

ISSN : 2032-0442

Éditeur

Institut de sociologie de l'Université Libre de Bruxelles

Édition imprimée

Date de publication : 27 juillet 2012

Pagination : 103-118

ISSN : 0009-8140

\section{Référence électronique}

Katerina Seraidari, «L' Homo Balkanicus en contexte migratoire », Civilisations [En ligne], 60-2 | 2012,

mis en ligne le 27 août 2012, consulté le 01 mai 2019. URL : http://journals.openedition.org/

civilisations/3027 ; DOI : 10.4000/civilisations.3027 


\title{
L'Homo Balkanicus en contexte migratoire
}

\author{
Un modèle d'identification à défendre ou à rejeter?
}

\author{
Katerina SERAÏDARI
}

Résumé : Basé sur des enquêtes de terrain menées auprès de Grecs et de Turcs de première et de seconde génération qui vivent actuellement à Bruxelles, cet article examine pourquoi les Turcs s'identifient davantage de façon positive aux Balkans, tandis que les Grecs donneraient plutôt une réponse négative. Si pour ces derniers, l'identité balkanique est une option possible parmi d'autres (comme l'identité européenne ou la référence à une spécificité méditerranéenne), dans le cas des Turcs, la référence balkanique semble constituer un modèle d'auto-présentation particulièrement valorisant. Le but est donc de comprendre comment les membres de ces deux groupes nationaux définissent leur position dans les Balkans afin de revendiquer un statut de supériorité. Les manipulations stratégiques des repères identitaires qu'effectuent ces acteurs sociaux révèlent leur manière de se positionner dans une construction spatiale définissant le centre et la périphérie de l'Europe.

Mots-clés : immigration, domination, Grèce, Turquie, Belgique.

\begin{abstract}
Drawing on field research conducted among Greek and Turkish migrants and their descendants living in Brussels, this article examines why people recognizing themselves as Turkish are more likely to identify positively with the Balkans, whereas people of Greek descent do not. For the latter, the Balkan identity exists as an option among others (as the claim to an European identity or a Mediterranean one), while for people of Turkish descent, the Balkan reference is more directly valued. In both cases, the strategic manipulations of identity references reveal how these social actors position themselves vis-à-vis Europe and define its centre and periphery.
\end{abstract}

Keywords: immigration, domination, Greece, Turkey, Belgium. 


\section{La Belgique a conclu une convention d'immigration avec la Grèce en 1957 et} avec la Turquie en 1964. C'est en 1955 que les premiers ouvriers grecs, destinés à travailler dans les mines, commencent à arriver sur le territoire belge : 10.195 Grecs reçoivent un permis de travail entre 1955 et 1961 (Martens 1976 : 101). En tant que « premiers arrivés » dans les régions minières par rapport aux Turcs, les Grecs, qui maîtrisent déjà mieux la langue du pays et qui ont eu le temps de s'initier au métier de mineur et aux codes culturels autochtones, ont tissé dès le début avec les Turcs des liens de solidarité qui avaient pourtant un fort aspect hiérarchique. Ce sont les mineurs grecs, qui connaissent un certain nombre de mots turcs (soit parce qu'ils viennent de régions grecques où ils cohabitaient avec les Turcs, comme en Thrace occidentale, soit parce que leurs parents étaient originaires d'Asie Mineure) qui ont initialement servi de traducteurs pour les Turcs. Ainsi, soutenus par les normes de l'ethnostratification qui classent les différents groupes d'immigrés selon leur ordre d'arrivée et étant acceptés comme « culturellement plus proches » que les Turcs par la société belge ${ }^{1}$, les Grecs ont été placés, dès le début, dans une situation plus confortable.

Selon Anastasia Antiochos (2002-2003 : 50-53), plus d'un tiers de ces immigrés grecs étaient originaires de Macédoine, tandis que dans les années 1962-1964, la Thrace occidentale $^{2}$ devient la première région fournissant cette main-d'œuvre. Il faut savoir que des réfugiés grecs, qui ont quitté la Turquie en 1922, se sont massivement installés dans ces régions frontalières grecques, dans le cadre d'une politique sécuritaire de l'État grec - ce qui explique pourquoi leurs descendants qui ont immigré en Belgique portaient souvent la mémoire d'un passé commun et, dans une grande mesure, d'une coexistence paisible dans le cadre de la pax ottomana ${ }^{3}$. L'émigration en Belgique

1. Selon la Statistique des étrangers, il y avait 602 Grecs de plus de quinze ans installés dans l'agglomération bruxelloise déjà en 1938, mais aussi 210 Yougoslaves et 753 Roumains. Dans le recensement des commerçants étrangers à Bruxelles-ville en 1939, il y avait 20 commerçants grecs, 16 commerçants roumains et 12 commerçants turcs (Taschereau, Piette et Gubin 2001 : 15 et 27). Dans le cadre de cet article, l'ordre d'arrivée de cette première vague d'immigration balkanique ne sera pas analysé. En revanche, l'ordre de la deuxième vague (assurant de la main-d'oeuvre pour les mines) donne clairement à voir une prééminence des Grecs, qui sont les premiers dans les Balkans à bénéficier de conventions bilatérales avec la Belgique. D'autre part, dans les livrets réalisés à la demande du ministère de l'Emploi et du Travail belge afin d'attirer une main-d'œuvre turque dans les années 1960, les Turcs sont caractérisés comme «travailleurs méditerranéens » (brochure citée par Parthoens et Manço 2005 : 35). Il s'agit ici de la volonté des autorités belges de souligner la continuité d'une politique de recrutement (après les Italiens, les Espagnols et les Grecs, les Turcs) et aussi d'encourager l'intégration des nouveaux venus dans cette « famille méditerranéenne ».

2. Cette région grecque abrite une importante communauté turque protégée par le Traité de Lausanne (1923) - texte régulateur du statut des populations non échangeables entre la Grèce et la Turquie.

3. Si une partie importante des migrants grecs ont manifesté, dès le début, leur solidarité envers les migrants turcs en Belgique, un certain nombre d'entre eux continuent de les considérer comme les représentants d'un peuple ennemi. Comme je l'ai montré ailleurs (Seraïdari 2011), trois postures comportementales définissent les rapports entre Grecs et Turcs en Belgique : hostilité, affinité, indifférence. Ces postures dépendent des circonstances et des contextes, mais aussi des intérêts et des positions idéologiques des individus. 
a suscité des discussions en Grèce, parce qu'un grand nombre de migrants grecs venaient précisément des régions frontalières (avec la Turquie ou les pays appartenant à l'époque au bloc de l'Est) et leur départ pouvait fragiliser l'économie locale et poser des problèmes d'ordre sécuritaire.

Les immigrés grecs qui viennent en Belgique prennent la décision de migrer soit en raison de leur pauvreté, soit pour faire face à des contraintes sociales (nécessité de constituer la dot de ses sœurs ou de ses filles, règles d'héritage morcelant la terre familiale). Dans les années 1950, ces régions frontalières souffrent d'un sousdéveloppement économique dû aux ravages de la guerre et à la fermeture des frontières avec les pays voisins étant passés dans le camp communiste. De plus, à partir de la guerre civile grecque (1946-1949) et jusqu'à la fin de la dictature (qui a duré de 1967 à 1974), ceux qui sont considérés comme " communistes » sont persécutés et subissent des discriminations qui leur rendent la vie impossible ; cette étiquette de « communiste » n'a jamais un caractère individuel, mais affecte la vie de tous les parents proches. Un certain nombre d'entre eux décide donc de quitter la Grèce pour des raisons politiques.

Les immigrés qui sont venus à Bruxelles ont voulu valoriser la partie la plus prestigieuse de leur passé national : la Grèce ancienne pour les Grecs, l'Empire ottoman pour les Turcs. L'évocation de l'héritage antique dont les Grecs se présentent comme les gardiens, a certainement facilité leur intégration dans le pays d'accueil. Le fait que l'Antiquité grecque soit considérée comme la base de la civilisation occidentale inscrit les Grecs dans la «grande famille » européenne ${ }^{4}$. En revanche, pour les immigrés turcs, la valorisation de l'Empire ottoman renvoie à l'espace balkanique. En effet, la référence balkanique prouve la présence multiséculaire des Turcs en Europe : leur rapport avec l'Europe passe par ce modèle d'identification. Même si après le Congrès de Berlin de 1878 le démantèlement de la « Turquie d'Europe » est dans une large mesure achevé, au début du XXe siècle, Gaston Gravier (1913) continue de parler de l'Albanie comme d'une partie de cette Turquie d'Europe. Cette tendance érudite ne peut pas être dissociée de la pratique vernaculaire balkanique d'utiliser le terme "Turc », encore aujourd'hui, pour désigner les musulmans des Balkans. Jean-François Gossiaux (2002 : 28) parle d'une « fusion ethnonymique des identités musulmane et turque $»$.

En m'appuyant sur des données que j'ai recueillies lors d'enquêtes de terrain auprès de Grecs et de Turcs de première et de seconde génération qui vivent actuellement

4. La lutte d'indépendance contre l'Empire ottoman, qui a suscité un courant philhellénique en Occident, constitue un autre facteur à prendre en considération. Dans l'imaginaire européen, l'hostilité présumée des Grecs envers les Turcs vient des «quatre siècles d'obscurantisme et d'esclavage » que les premiers ont vécus sous le joug d'un peuple non chrétien après la chute de Constantinople (la « deuxième Rome »). En fait, le philhellénisme occidental des XVIII et XIX ${ }^{\mathrm{e}}$ siècles est surtout lié à l'admiration pour l'Antiquité grecque : les récits des philhellènes qui visitent la Grèce reflètent leur déception quand ils découvrent que les Grecs modernes n'ont pas grand chose à voir avec les Grecs anciens. Les philhellènes considèrent ces voyages comme une sorte de pèlerinage capable de les transférer non seulement dans un autre espace mais aussi dans un autre temps (l'Antiquité). 
à Bruxelles 5 , j'analyserai pourquoi les Turcs semblent répondre positivement à l'alternative " être ou ne pas être balkanique », tandis que les Grecs donneraient plutôt une réponse négative. L'objectif est de comprendre pourquoi l'identification comme Homo Balkanicus serait plus légitime pour les uns que pour les autres. Car si pour les Grecs l'identité balkanique est une option possible parmi d'autres (comme l'identité européenne ou la référence à une spécificité méditerranéenne), dans le cas des Turcs, la référence balkanique semble constituer un modèle d'auto-présentation particulièrement valorisant. Même si l'immigration turque provient dans sa grande majorité d'une seule région, la sous-préfecture d'Emirdag dans la province centreanatolienne d'Afyon, et que le bas du quartier bruxellois de Schaerbeek est souvent appelé « la petite Anatolie », l'héritage balkanique est bel et bien revendiqué. Comme un informateur turc l'a dit à Christina Moutsou (1998 : 195-196, note 179), « Tout dépend de ce que le Turc veut. S'il veut être considéré comme un musulman, alors il se pense proche des Arabes. Mais s'il veut être vu comme un européen, alors il reconnaît des similitudes culturelles avec les Grecs ». Si l'accès à l'identité européenne est lié, pour les Turcs vivant en Belgique, à la mémoire de l'Empire ottoman qui a dominé une partie de l'Europe, pour les Grecs, cette même référence est celle qui pose problème : les similitudes culturelles avec les Turcs ne renforcent pas le caractère européen des Grecs, bien au contraire, elles en montrent les limites.

En fait, l'adhésion ou non au modèle de l'Homo Balkanicus ne se pose pas en termes d'appartenance (à un territoire déterminé par la géographie et l'histoire) pour les Grecs et les Turcs, mais en termes de rapports de force. Le but est donc de comprendre comment les membres de ces deux groupes nationaux dans le contexte migratoire belge définissent leur position dans les Balkans afin de revendiquer un statut de supériorité. Comme nous le verrons, les manipulations stratégiques des repères identitaires qu'effectuent ces acteurs sociaux révèlent leur manière de se positionner dans une construction spatiale définissant le centre et la périphérie de l'Europe.

\section{Des produits et des hommes : processus de nomination et de domination}

Quand j'ai interrogé deux informateurs turcs de la seconde génération (originaires d'Emirdag et membres d'une Association turque) sur la longue coexistence des Grecs et des Turcs dans le quartier bruxellois de Saint-Josse, ils ont fait référence aux premiers commerces vendant des produits qu'ils considéraient comme « balkaniques » (feta, olives, poivrons, concentré de tomates) dans les années 1970 : les commerçants étaient tous grecs, et les immigrés turcs qui sont venus s'installer dans ce quartier sont immédiatement devenus leurs clients. Leur récit illustre la proximité culturelle des Grecs et des Turcs - définie par des modèles communs de consommation et le partage de codes culturels similaires. Pourtant, aucun Grec n'aurait défini les olives

5. Enquêtes effectuées entre octobre 2010 et septembre 2011 dans le cadre du projet « Quand deux insularités socio-culturelles se côtoient au cœur de l'Europe : le rapport à l'intégration des communautés grecque et turque de Bruxelles et leurs relations mutuelles », financé par l'Institut d'encouragement de la Recherche Scientifique et de l'Innovation de Bruxelles (IRSIB, Convention « Brains to Brussels ») et sous la supervision d'Ural Manço (Facultés universitaires Saint-Louis). Je voudrais remercier Ural Manço pour sa collaboration stimulante lors de cette recherche, et Galia Valtchinova pour ses commentaires sur une version précédente de cet article. 
et, surtout, la feta, comme un « produit balkanique ». Il est intéressant de juxtaposer ce témoignage avec celui d'une informatrice grecque (installée à Bruxelles depuis 1982) : elle achète ses fruits dans une épicerie turque qui se trouve dans le quartier de Woluwe-Saint- Lambert, ce qui constitue son seul rapport avec un membre de cette communauté. Ce commerce vend « des fromages grecs, des olives, de l'aneth et de la laitue, des betteraves qui ont encore leurs feuilles ", et beaucoup de Grecs vont y faire leurs courses, on y fait même la queue. Comment interpréter le fait que les épiceries grecques du quartier populaire de Saint-Josse vendaient dans les années 1970 de la feta (« produit des Balkans ») et qu'une épicerie turque du quartier aisé de Woluwe vend actuellement des «fromages grecs »? Si dans le premier cas, nous sommes dans un contexte ouvrier, dans le second, ce commerce vise à satisfaire les besoins alimentaires d'une clientèle grecque fortunée - qu'elle soit descendante de mineurs qui ont connu une ascension sociale et par conséquent, se sont déplacés vers la périphérie de la ville, ou des « Eurocrates » installés au « cœur de l'Europe » depuis l'entrée de la Grèce dans l'Union européenne en 1981. Il existe très peu d'épiciers grecs actuellement à Bruxelles, ce qui montre que ces immigrés et leurs descendants ont pu s'orienter vers des métiers plus lucratifs et moins contraignants ; en revanche, l'épicier turc constitue presque un stéréotype (correspondant à celui de l'« épicier arabe » en France) révélant le dynamisme commercial de cette communauté, qui a connu dans sa majorité une ascension sociale moins importante que celle des Grecs. Mais comment expliquer cette distinction dans la manière de nommer le fromage («balkanique » ou « grec ») ?

Pour traiter cette question, il faut sortir du contexte migratoire et examiner brièvement la politique que l'État grec a adoptée dès les années 1980 en ce qui concerne la protection de la feta comme produit national grec. Le 12 juin 1996, la Communauté européenne a accordé l'AOP (Appellation d'origine protégée) à la feta. Néanmoins, l'Allemagne, le Danemark et la France ont déposé un recours devant la Cour de justice européenne contre cette décision, "soutenant que le mot 'feta' était une 'dénomination générique' et désignait une technique de production très courante d'un fromage fabriqué dans tous les pays balkaniques sous des appellations différentes » (Petridou 2005 : 257). Début 2003, la protection de la feta a été réaffirmée par les institutions européennes, argumentant en faveur « de la grécité de la feta » qui serait produite " de manière attestée en Grèce depuis l'Antiquité » (ibid). Petridou cite certaines publications grecques ayant pour but de prouver que « la préparation d'un fromage 'frais' à partir de lait de chèvre et de brebis existe, entre autres, dans l'Odyssée d'Homère, dans les Cavaliers d'Aristophane et chez Aristote » (ibid : 259). Enfin, Petridou analyse comment cette « bataille du nom » rejoint un autre problème national qui a surgi dans les années 1990 : avec l'éclatement de la Yougoslavie émerge un nouvel État qui souhaite se nommer « République de Macédoine »-ce qui provoque une vive réaction de la part de la Grèce qui impose au niveau international le nom FYROM (Former Yugoslav Republic of Macedonia) ${ }^{6}$. Cette affaire est aussi

6. Même dans les productions académiques, les mots employés comptent et sont investis d'une dimension politique. Le livre édité par Gilles de Rapper et Pierre Sintès (2008) commence par cette remarque liminaire : «Aussi, dans trois textes du volume, l'École française d'Athènes a-t- 
liée à la question de la grécité d'Alexandre le Grand ${ }^{7}$ et des Macédoniens anciens. Dans les deux cas, l'expression « bataille du nom » esquisse « un système de pensée qui traverse ces deux événements et se concentre sur l'idée de la menace et de la revendication nationales » (ibid : 261). Dans cet article, Petridou se réfère à une autre affaire qui a préoccupé l'opinion grecque dans les années 1990, le retour des marbres du Parthénon. La mise en parallèle de ces trois controverses est intéressante, même s'il s'agit chaque fois de registres différents : dans le cas de la feta, nous sommes dans le registre d'une concurrence marchande régulée par les instances européennes; dans le cas de la Macédoine, dans le registre politique et diplomatique ${ }^{8}$; et dans le cas des marbres du Parthénon, dans le registre patrimonial. De plus, les rapports de force qui sont distincts selon chaque registre ne sont pas analysés dans cet article, la Grèce se positionnant toujours comme victime d'usurpation et de dépossession, mais tenant un rôle dominant dans la controverse sur la Macédoine (ce qui n'a pas été le cas dans les deux autres affaires).

La question de la Macédoine constitue, pour certains Grecs, une controverse simplement onomastique et donc surévaluée, tandis que pour d'autres, il s'agit d'un enjeu politique (voire géopolitique) majeur'. Tant pour la Macédoine que pour la feta, ce qui se dessine est la défense de la spécificité grecque contre l'acceptation d'un passé balkanique commun. Le recours à la Grèce antique sert à assurer des droits de propriété sur le territoire, ses populations, ses produits, sa continuité historique, son patrimoine culturel, ses noms. Autrement dit, « ce qui est à nous ne peut pas être balkanique ", car dans ce cas, la question d'une appropriation possible serait posée, et cela serait contre les intérêts nationaux grecs. En mettant l'accent sur le temps et sa profondeur, la référence antique finit par extraire la Grèce de l'espace balkanique, considéré comme une construction postérieure ; cette référence définit non seulement l'exceptionnalité grecque, mais efface aussi toute revendication possible de la part des voisins, en créant un «monopole historique ». Ainsi, l'acceptation d'une identité balkanique pour les Grecs est assimilée à un geste de concession et d'abandon de ce

elle demandé aux auteurs de remplacer l'appellation de 'République de Macédoine', adoptée par les autorités et les habitants du pays en question, par celle d'AAncienne République Yougoslave de Macédoine', seule reconnue à ce jour par la France, l’Union européenne et l'ONU ».

7. Un informateur grec de la première génération, qui est né en 1943 dans une ville de Macédoine (près de Pella) et est venu en Belgique en 1965, m’a expliqué qu'Alexandre le Grand était motivé par le projet d'unir les peuples et ne voulait pas les conquérir comme Napoléon ou Hitler. Il s'agit d'un schéma rhétorique courant en Grèce, qui réfute tout effort de conquête ou d'oppression des peuples étrangers dans le cours de l'histoire grecque. Cet informateur m'a aussi dit que si les réfugiés grecs ne s'étaient pas installés en Macédoine après l'échange des populations, la Macédoine serait maintenant aux mains des Slaves, des Bulgares et des Turcs.

8. Commentant la «nouveauté absolue » d'« un copyright sur les noms géographiques » que les Grecs ont introduite dans le droit international, Paul Garde (2004 : 210) montre pourtant que pour soutenir leur position, les Grecs « se réfèrent parfois au droit commercial, au fait que 'champagne' est une appellation contrôlée ». Dans le cadre de cette affaire, il semble que les deux registres (droit international et droit commercial) aient bel et bien été mélangés.

9. La Turquie a été le premier pays à reconnaître officiellement la « République de Macédoine » le 6 février 1992 (Alexandris 1995 : 501). Alexandris soutient que le seul point de convergence et de coopération entre la Turquie et le FYROM est leur opposition à la Grèce (ibid : 504-505). 
qui est « à nous » au bon vouloir d'éventuels rivaux. Comme nous l'avons vu, dans le cadre d'une concurrence commerciale, l'Allemagne, le Danemark et la France ont eu recours à l'argument de la « provenance balkanique » de la feta afin de se poser en adversaires de la « juste cause grecque ». Ces pays constituaient alors des acteurs probablement plus redoutables que les potentiels rivaux balkaniques, à cause de leur puissance économique et de leur poids géopolitique. La mise en doute de la grécité passe, ici aussi, par la référence balkanique : « ce qui est balkanique ne peut pas être à vous ». Comme nous l'avons vu plus haut, cette équivalence ne se pose pas dans ces termes dans la rhétorique nationale turque, où « ce qui est balkanique est aussi turc ». Pour le dire autrement, à la référence antique au caractère exclusif se juxtapose la référence inclusive à l'Empire ottoman.

Retournons maintenant au contexte migratoire belge. Étant donné qu'un grand nombre d'immigrés grecs à Bruxelles viennent de la Macédoine grecque, il n'est pas étonnant que des manifestations y aient été organisées afin de défendre la grécité de la Macédoine (dont l'intégrité territoriale, selon leur lecture des faits, était menacée). Plusieurs informateurs grecs m'ont parlé de leur mobilisation contre les prétentions des « faux Macédoniens » de FYROM. C'est en interviewant une Grecque, originaire de Thrace, qui s'est installée à Bruxelles en 1987 afin d'aider son frère qui dirigeait un snack au centre de Bruxelles, que j'ai réalisé que les avis n'étaient pourtant pas unanimes. Elle m'a parlé de son beau-père, qui n'était pas d'accord avec les idées de ceux qui fréquentaient l'Association des Macédoniens à Bruxelles, parce qu' « ils ont mis la politique dedans » (evalan mesa tin politiki). Puis, elle a mentionné un militaire grec impliqué dans le conflit macédonien au début du $\mathrm{XX}^{\mathrm{e}}$ siècle, Pavlos Melas ${ }^{10}$ (1870-1904), dont l'action militaire a visé à assurer le rattachement de la région à la Grèce : «Les 'autochtones' (dopioi) parlent de manière négative de Pavlos Melas. Il a nui aux villages et à ceux qui n'étaient pas d'accord avec ses idées. Dans le village de mon beau-père, près des frontières, il a laissé de mauvais souvenirs ». Elle m'a expliqué que lors de la période de mobilisation sur la « question de la Macédoine » dans les années 1990, son beau-père ne savait pas de quel côté se mettre :

\section{Il disait qu'il se sentait plus proche de Skopje que d'Athènes. Si l'État de Skopje avait été plus organisé, il aurait préféré être de ce côté-là. Pas seulement lui, mais tous ceux qui habitent dans cette région frontalière. Ils se sentent lésés (adikimenoi), parce que les Athéniens présentent l'histoire d'Alexandre le Grand comme ils veulent. Mon beau-père était macédonien, il se sentait grec, mais il était en désaccord avec tout ce que les Athéniens voulaient enseigner sur la Grèce du Nord, des choses comme cela. Il pensait que la Grèce du Nord était délaissée.}

Ce récit met en place une opposition entre "Athéniens » (privilégiés et représentants du pouvoir décisionnel) et habitants de la Grèce du Nord (délaissés, muets et donc obligés de reproduire le discours athénien). J'avais déjà recueilli en

10. Sur Pavlos Melas, qui était le fils éduqué et cosmopolite d'une famille influente de la bourgeoisie athénienne, voir Karakasidou (2004). Karakasidou souligne « la sophistication urbaine dans l'imagerie de Melas » qui contraste avec la « rusticité indisciplinée » de ses opposants pro-bulgares (ibid : 209). 
Grèce dans les années 1990 des témoignages allant dans ce sens : un jeune homme de Thessalonique m'avait même dit que c'était les commerçants athéniens qui avaient « fabriqué cette histoire de Macédoine » pour empêcher le développement économique de Thessalonique, pour laquelle de nouvelles perspectives s'ouvraient après l'effondrement du rideau de fer. Premier constat : les récits recueillis en Grèce et dans le contexte migratoire belge sont, dans ce cas, similaires. Ces témoignages suggèrent qu'à la question « être ou ne pas être balkanique ", les " Athéniens » (le héros national Pavlos Melas étant classé dans cette catégorie par certaines personnes) et les habitants de la Grèce du Nord ne peuvent pas donner la même réponse, à cause de leurs expériences et de leur positionnement différent dans l'espace géographique et social. Sans être représentative de la grande majorité de la population grecque, cette position est défendue non seulement par la minorité slavophone grecque (dont le beau-père de mon informatrice faisait partie), mais aussi par ceux qui condamnent les mesures discriminatoires prises par l'État grec contre cette minorité depuis le rattachement de la région et qui défendent la vision d'une société grecque pluraliste.

L'analyse géopolitique de Georges Prévélakis (2002 : 138), qui soutient que « jusqu'en 1989, le rideau de fer protégeait la Grèce de son passé », nous permettra de passer des récits ethnographiques au discours académique. Après avoir souligné le fait que « la fin de la guerre froide a mis la Grèce en contact avec son histoire balkanique », Prévélakis constate que « la Grèce se comporta envers la Macédoine comme la Grande-Bretagne et la France s'étaient comportées envers la Grèce pendant le $\mathrm{XIX}^{\mathrm{e}}$ et le début du XXe siècle. Le blocus de la Macédoine par Athènes entre 1994 et 1995 s'inspirait des méthodes de l'impérialisme occidental » (ibid : 138-139).

L'article de Prévélakis laisse le lecteur perplexe, dans la mesure où il considère que les Grecs, au lieu de devenir les leaders européens des Balkans et les représentants des intérêts balkaniques en Europe, se sont laissés entraîner dans la « balkanisation ». Cette analyse pose la Grèce, à la fois, à l'intérieur et en dehors des Balkans : elle aurait pu jouer un rôle hégémonique dans le but de gérer cet environnement balkanique et de promouvoir son intégration à l'Europe (afin de mieux servir ses propres intérêts nationaux). Mais, en fin de compte, elle a été rattrapée par sa « nature balkanique », puisque ses défauts « structurels » ont émergé, montrant qu'elle n'était pas capable d'assumer cette ambition « civilisatrice »: nationalisme, corruption, clientélisme, « désagrégation morale, politique et économique », « effondrement de la gouvernance» (ibid : 143). La contradiction se trouve dans la distinction entre un «bon » et un « mauvais » rôle hégémonique : la Grèce aurait pu prendre exemple sur ses partenaires européens (qui n'utilisent plus les " méthodes de l'impérialisme occidental »), mais son attitude envers la Macédoine a été anachronique et arrogante, puisqu'elle s'est avérée être une mauvaise imitatrice de pratiques occidentales désuètes.

Ce qui nous intéresse ici sont les rapports de domination que la référence à l'Homo Balkanicus fait surgir : d'abord à une échelle européenne, entre un «bon » et un " mauvais » rôle hégémonique (le premier définissant les Européens et leur leadership politico-économique, le second, ceux qui ne peuvent pas échapper à leur héritage historique, voir au déterminisme de leur " nature balkanique ») ; puis, à une échelle nationale, entre «Athéniens » (dominants) et habitants de la Grèce du Nord (dominés), ou entre les « vrais » et les « faux » Macédoniens. L'analyse géopolitique 
de Prévélakis se focalise sur la dimension européenne de la question, tandis que pour les informateurs grecs (de Grèce et de Belgique), l'accent est surtout mis sur les enjeux nationaux. Si l'appropriation du passé de la Grèce antique par l'Occident a pu prendre la forme d'une rhétorique auto-valorisante (« nous, les Grecs, sommes à l'origine de la civilisation occidentale »), les revendications de ce passé par un autre pays balkanique ${ }^{11}$ sont perçues comme menaçantes et comme un danger potentiel pour l'intégrité territoriale de la Grèce. Si l'héritage antique exclut l'existence d'une réalité balkanique tout en s'inscrivant dans une logique européenne, la référence à l'Empire ottoman fonctionne de manière inverse : elle crée, par elle-même, l'espace balkanique sans pouvoir pourtant s'inscrire dans une « centralité européenne ».

Selon l'importance géopolitique de l'acteur et ses intentions prétendues, on fait donc la part des choses entre une « bonne » et une « mauvaise » manière de clamer la participation au prestigieux héritage de l'Antiquité. Si dans le premier cas, il s'agit d'un « jeu gagnant » qui reconnaît la contribution de la Grèce à l'histoire de l'Humanité, dans le second, c'est un « jeu perdant» qui pourrait coûter à la Grèce la perte d'une partie de son territoire. Derrière cette grille de lecture, il y a l'idée que les " grands » pays tracent le cours de l'histoire de l'Humanité, tandis que les « petits » pays (comme ceux qui se trouvent dans les Balkans) ne s'intéressent qu'à leurs " petits » intérêts et succombent aux affrontements interethniques; les premiers sont ainsi positionnés en permanence du côté de l'universalisme, et les seconds du côté du nationalisme ${ }^{12}$. De ce point de vue, la Grèce veut être reconnue comme un « grand » pays - par son histoire et sa place dans la « grande famille » européenne. Mais chaque fois qu'elle

11. Selon Georges Castellan (2003 : 45), la référence à Alexandre le Grand fait son apparition dans les populations slaves de cette région dans les années 1840 : les voyageurs russes qui traversent le pays sont frappés par la popularité de ce personnage historique « dans les pesni des chanteurs autochtones ». Kyril Drezov (1999: 50) cite le témoignage du voyageur et universitaire russe Victor Grigorovich, qui a remarqué en 1845 que la popularité exceptionnelle d'Alexandre le Grand semblait avoir été imposée à la population, qui ne pouvait décrire ce héros sans se référer à son enseignement scolaire. Drezov considère que « la présence massive d'écoles et d'enseignants grecs a conduit inévitablement à une certaine 'Macédonisation' de tous les Chrétiens orthodoxes, même de ceux qui n'étaient pas Grecs d'un point de vue ethnique et linguistique » (ibid). Selon cette analyse, les couches dominantes grecques du XIX siècle ont produit et diffusé une idéologie, qui est devenue par la suite une des sources de l'éveil national de la population slave.

12. Bien sûr, ce schéma s'est renforcé dans les années 1990. En revanche, pendant la guerre froide, ceux qui prônaient les mérites du régime socialiste accusaient les pays capitalistes (comme l'Irlande, la Belgique, ou la France et l'Espagne à propos de la gestion de la question du Pays basque) d'être impliqués dans des situations d'affrontement durable allant parfois jusqu'à la violence, tandis que les pays socialistes ne souffraient pas de ce type de problèmes (Roux 1992 : 29). Dans ce cadre, l'universalisme marxiste s'opposait au système capitaliste qui engendrait des conflits nationalistes, les luttes nationales cachant l'importance des luttes de classe. De même, une des idées du mouvement turc des fethullahci, qui s'est implanté en Macédoine (FYROM) dans les années 1990, est que l'unité islamique, qui « passe par la revivification du monde turc », a été corrompue par le nationalisme, ce « feu jeté sur le monde musulman depuis les laboratoires d'idées de l'Occident » (Clayer 2001 : 205). Ce type de discours présente non seulement le nationalisme comme un «mal importé », mais aussi le communisme comme une source de souffrance qui a surtout pénalisé les musulmans des Balkans. La manière de définir le nationalisme dans les Balkans a toujours été liée à des clivages idéologiques (communisme versus capitalisme, Orient versus Occident, Christianisme versus Islam). 
s'implique dans des controverses politiques comme celle de Macédoine, elle perd ce droit de « grandeur », révélant son incapacité à sortir de la logique supposée dominer dans les pays balkaniques.

Pour revenir au contexte migratoire qui est au centre de cette analyse, le témoignage sur le migrant de nationalité grecque, qui ne savait pas comment se positionner entre l'État grec et « l'État de Skopje » (comme celui-ci est nommé en Grèce) auquel il était lié par une allégeance affective ${ }^{13}$, montre que les « Grecs » et les « Turcs » dont il est question dans cet article sont des termes génériques qui tendent à occulter toute fracture dans la supposée homogénéité de la population de ces nations. Lors de mes interviews, certains informateurs turcs ont utilisé des termes comme ceux de TurcoAlbanais et de Turco-Macédoniens ${ }^{14}$. Ils m'ont affirmé que les Turco-Albanais qui vivent à Bruxelles sont plus progressistes que les autres membres de la communauté turque : souvent affiliés à la gauche, ils se soucient de l'éducation de leurs enfants. Les Turco-Macédoniens auraient également mieux réussi leur vie en Belgique. Bien sûr, les termes Turco-Albanais et Turco-Macédoniens peuvent être interprétés comme des efforts afin de « capter » l'allégeance de certaines populations musulmanes des Balkans, qui seraient restées fidèles à une supposée mère patrie turque. De ce point de vue, tant l'existence (dans le cas de la Turquie) que l'absence (dans le cas de la Grèce) de ce type de désignations peuvent être également perçues comme des tentatives de prise de contrôle identitaire ${ }^{15}$.

\section{L'enjeu des catégorisations}

Un informateur grec de la seconde génération, qui est propriétaire d'un restaurant grec à Uccle, m'a parlé avec admiration du restaurant L'Athénien, dans la Chaussée de

13. Sa belle-fille, originaire de Thrace, m’a expliqué que dans sa propre région natale ce type de questions ne se pose pas : tous sont unis contre celui qui est considéré comme une menace potentielle, le Turc. Néanmoins, elle est consciente du dilemme de son beau-père, qui était exposé à deux cultures : tous les gens de son village parlaient le dialecte slave local entre eux et n'apprenaient le grec qu'à l'école. Son mari ne parle pourtant pas ce dialecte, il le comprend seulement ; ayant grandi à Bruxelles, cette transmission a été interrompue en faveur de l'apprentissage du français, lors de sa scolarité, et du grec.

14. La rupture entre Tito et Staline, qui a eu lieu en juin 1948, a conduit Belgrade à signer avec la Turquie et la Grèce des traités de coopération et d'amitié en 1953 (Castellan 2003 : 79). Dans les années qui suivent, la Yougoslavie titiste autorise les Turcs et autres musulmans vivant sur son territoire à émigrer vers la Turquie ; une partie d'entre eux est ensuite venue en Belgique. Michel Roux (1992 : 155, note 20) estime cette émigration en Turquie aux environs de 139000 personnes pour la période 1953-1961, et à 34000 personnes entre 1961 et 1971.

15. À l'inverse, les acteurs sociaux manipulent aussi ces ouvertures identitaires turques afin d'améliorer leur condition de vie ou, dans le cas de l'ex-Yougoslavie, afin de pouvoir profiter de la possibilité d'émigrer en Turquie. Avant 1966, en Yougoslavie, « l'identité 'turque' était très en faveur, c'était un moyen pour les albanophones d'échapper à bien des désagréments » (Garde 2004 : 156). Mais après l'éviction de Rankovic, chef du service de sécurité de l'État, qui avait introduit une politique de répression contre les Albanais (conséquence de la rupture Belgrade-Tirana qui a suivi la rupture Moscou-Belgrade en 1948), « le nombre des 'Turcs' décrut soudain dès le premier recensement, celui de 1971, et de mauvais plaisants parlèrent de 'génocide statistique des Turcs'» (ibid). Sur les processus de « mimétisme ethnique » (ethnic mimicry) et les systèmes de « patronage ethnique » (ethnic patronage) en ex-Yougoslavie, voir Duijzings (2000 : 147-156). 
Louvain, qui a ouvert au début des années 1980. Selon lui, cet établissement marque l'apogée de l'engouement pour ce type de "folklore grec » combinant l'image du « bon vivant» (selon le stéréotype hollywoodien de Zorba le Grec) et celle de l'héritier de la Grèce antique : les Belges faisaient la queue pendant deux heures pour manger à L'Athénien et voir le décor, « avec les statues, c'était comme un temple grec ». Ce succès a duré dix ans, jusqu'à la mort précoce du propriétaire, à l'âge de trente-cinq ans. L'artiste qui a créé ce décor antique était « un Grec de Macédoine, d'origine slave » : « Il ne faisait pas de tableaux ni de dessins. Il faisait des colonnes, des décors en bois, des reliefs ». Un peintre grec qui est arrivé à Bruxelles en 1985 et a décoré plus de 500 snacks, cafés et restaurants, dont la grande majorité à Bruxelles (mais aussi en Grèce, en Hollande et ailleurs en Europe), m'a aussi parlé de son prédécesseur :

Dans les années 1970, le seul qui s'occupait de la décoration des restaurants grecs était un Yougoslave, qui peignait surtout des batailles évoquant la Grèce antique, avec des javelots et des glaives. Mais celui qui va au restaurant pour manger, ne veut pas voir du sang qui coule. Il faut des thèmes plus romantiques, plus appropriés.

Il est vraisemblable qu'il s'agisse du même artiste, désigné par un informateur comme « Grec de Macédoine, d'origine slave » et par un autre comme « Yougoslave ». Il a donc mis ses talents de peintre et de décorateur au service des restaurateurs grecs afin de mettre en scène, pour le public belge, une imagerie de la Grèce antique que les migrants grecs ont su commercialiser. Comme pour les « fromages grecs » et la « feta balkanique ", ce qui n'est pas disputé peut être investi de modèles d'identification multiples. Mais dans un contexte de controverse, cette souplesse sémantique et cognitive fait place à une rigidité des revendications, où chaque partie met en avant ses preuves de paternité exclusive.

L'existence de groupes minoritaires dans la Grèce du Nord, comme les slavophones ou les Pomaques et les Turcs du Rhodope, a fait que le rideau de fer n'a jamais réellement coupé ces régions grecques de l'environnement balkanique. Une partie de la population avait gardé des contacts familiaux et des attaches avec ceux qui habitaient de l'autre côté de la frontière. Quand une femme de la seconde génération m'a raconté l'arrivée de son père (né en Thrace occidentale) en Belgique, elle a commencé par l'histoire de son grand-père paternel, qui était communiste et avait participé à la guerre civile grecque. Après la défaite de son camp, il était parti en Bulgarie, laissant derrière lui sa femme et son fils (le père de mon interlocutrice). Il s'y était remarié, avait adopté un enfant, mais n'avait pas voulu ajouter à son nom une terminaison slave, comme d'autres Grecs l'avaient fait ; cela ne l'avait pas empêché de monter dans la hiérarchie du Parti communiste bulgare et d'occuper un poste important. Du côté grec, la femme de cet exilé s'était aussi remariée et avait eu des enfants ; une fois devenu adulte, le père de mon interlocutrice, qui n'avait pas de bonnes relations avec son beau-père et qui « était inscrit sur la liste rouge, à cause de son père communiste », a voulu quitter le pays. Il est venu travailler dans les mines de Charleroi en 1964. Ayant peur d'être arrêté s'il retournait en Grèce, il n'a fait ce voyage que dix ans plus tard, après la chute de la junte. Entre-temps, le grand-père de Bulgarie était venu à Bruxelles pour voir son fils et ses petits-enfants élevés en Belgique. Ce récit révèle comment la Belgique est devenue pour certains Grecs une 
terre de refuge contre les persécutions politiques, et un lieu où des familles éclatées ont pu se retrouver après des années de séparation.

Si jusqu'à la Deuxième Guerre Mondiale, des conflits opposent les peuples balkaniques entre eux et leur imposent de choisir une affiliation nationale précise, à l'ère du monde bipolaire de la guerre froide et de la division des Balkans en deux camps opposés, le fait de revendiquer une identité balkanique n'est pas non plus courant. Jusqu'à l'effondrement du rideau de fer, la question « être ou ne pas être balkanique » se pose pour les Grecs et les Turcs dans les termes d' " être ou ne pas être communiste ». Pendant cette période, les Balkans sont une entité géographique discontinue, la Grèce et la Turquie appartenant au bloc de l'Organisation du Traité de l'Atlantique Nord, et les autres pays de la région au bloc du Pacte de Varsovie. Fendiye Kisacik (2000-2001 : 44) a trouvé dans la correspondance de la Fédéchar (Fédération des Charbonnages Belges engagée dans le recrutement de la main-d'œuvre pour les mines) des éléments concernant l'organisation du transport des migrants turcs : le chemin de fer impliquait la traversée de la Bulgarie et de la Yougoslavie, ce qui déplaisait aux organisateurs. Dans un document de la Fédéchar (22-27 avril 1963), on lit que « la Turquie a connu auparavant une malheureuse expérience avec les travailleurs pour l'Allemagne ». Kisacik rappelle que « ces deux pays communistes sont sous la tutelle de l'ex-Union Soviétique contrairement à la Turquie, qui se présente comme un pays capitaliste, en relation étroite avec les États-Unis. Le 'climat' de la guerre froide se fait sentir » (ibid : note 146). Bien sûr, la Yougoslavie n'est pas « sous la tutelle de l'Union Soviétique » dans les années 1960, et les formes de dépendance de la Bulgarie et de la Yougoslavie à l'égard de Moscou à cette époque ne sont pas du tout comparables. Mais cet extrait révèle la tendance générale à partager les pays balkaniques en deux blocs sans prendre en compte leurs spécificités et leur évolution politique $^{16}$. En ce qui concerne la première vague migratoire grecque, la situation est comparable :

L'ensemble du voyage s'effectuait par chemin de fer, via la Yougoslavie, l'Autriche et l'Allemagne. En vertu d'un accord passé entre la Fédéchar et les chemins de fer grecs, des trains spéciaux étaient affrétés par la Compagnie hellénique des Chemins de Fer jusqu'à Belgrade, où les wagons occupés par les migrants étaient accrochés au Tauern Express. (Antiochos 2002-2003 : 42)

Mais en août 1955, l'acheminement des migrants par Brindisi est décidé à cause d'incidents survenus avec des policiers yougoslaves. Selon la correspondance de la Fédéchar (31 mai 1956), « il arrive aussi, lorsque les places manquent, que les candidats recrutés par la Fédération soient tout bonnement expulsés du Tauern Express, au profit des Yougoslaves qui se rendent en Allemagne » (ibid : 43). L'arrangement avec les autorités yougoslaves peut donc être compromis par la concurrence sur le marché du travail. Le témoignage ${ }^{17}$ d'un mineur grec nous laisse entrevoir les craintes que la traversée de la Yougoslavie suscite :

16. Ainsi, même si la Grèce et la Turquie font partie toutes les deux du monde dit « libre », le conflit chypriote envenime leurs relations depuis les années 1960.

17. Theoharis Theoharis, né en 1937 en Macédoine et arrivé en Belgique en 1963. CD-Rom intitulé Mémoires d'Europe (Programme Socrates Grudtvig), produit par Nimrod asbl en 2006. 
"Le voyage m'a fait peur, le fait de sortir de nos frontières. Parce que la Yougoslavie était beaucoup plus arriérée que nous ici. Et je ne pouvais pas imaginer ce que je trouverais quand j'arriverais là-bas. Mais quand je suis arrivé en Autriche, mon cœur s'est ouvert. Je me suis dit, c'est bon, en avant!

Puis, à la question de savoir quels sont ses premiers souvenirs après son arrivée en Belgique, il répond : «Un endroit superbe, tout était tellement en ordre que nous ici ressemblions à la Yougoslavie » (itan toso taktopoïmeno pou emeis edo moiazame san tin Yougoslavia).

Si le départ vers l'Europe de l'Ouest dans les années 1950 et 1960 constitue une expérience qui met les migrants grecs et turcs en rapport avec ce qu'ils considèrent comme un «bloc de l'Est» homogène, les voyages que ceux-ci effectuent en voiture, en train ou en bus quelques années plus tard afin de retourner dans le pays d'origine pour passer des vacances s'inscrivent dans un autre registre, celui de la reconnaissance du fait d'appartenir à une culture commune. Ce récit peut être interprété de la façon suivante : si, dans un premier temps, le passage par la Yougoslavie confirme un préjugé récurrent, dans un deuxième temps, le séjour en Belgique révèle la ressemblance entre ce pays balkanique et la Grèce. Sortant du cadre du monde bipolaire et du clivage politique, la catégorisation entre " pays ordonnés » et " pays désordonnés » trace maintenant les limites entre l'Europe occidentale et les Balkans. Le contexte migratoire facilite cette mise à distance, ce regard détaché porté sur le pays d'origine. Le « stigmate du balkanisme » est dans ce cas évoqué pour valoriser la construction d'une nouvelle vie dans le pays d'accueil, et cela est valable tant pour les Grecs que pour les Turcs : le déplacement vers un pays plus « évolué » rend légitime la décision de partir et inscrit le migrant dans un mouvement évolutif non seulement en termes économiques mais aussi en termes d'organisation sociétale (de la confusion vers l'ordre) et d'importance géopolitique (de la périphérie vers le centre).

En 1986, lors d'un voyage d'Allemagne vers la Turquie, l'anthropologue Ruth Mandel (2008 : 238), qui accompagnait ses informateurs turcs, a constaté que leur attitude changeait au fur et à mesure qu'ils s'éloignaient de l'Europe occidentale. En arrivant dans les Balkans, ils insistent sur leur familiarité avec le paysage, les gens, la nourriture, l'architecture et l'ambiance générale. Un jeune informateur turc (petit-fils d'immigrés du côté maternel et fils d'immigrés du côté paternel) m'a fait la même remarque concernant le voyage en voiture que sa famille effectuait dans les années 1980 : chaque fois que le bateau arrivait d'Italie en Grèce, il avait l'impression d'être déjà en Turquie. Ici, la traversée des pays balkaniques suscite le sentiment rassurant d'être " chez soi », de faire partie d'une même aire culturelle et d'une même entité régionale. Si pour les migrants turcs les Balkans semblent être un espace transitoire conduisant de l'Europe de l'ouest à la Turquie, pour le Grec dont nous avons vu le témoignage plus haut, ils constituent plutôt une rupture spatiale, une mesure révélant l'incapacité de la Grèce à être comme l'Europe de l'Ouest.

\section{Conclusion}

Les Grecs et les Turcs de Bruxelles sont conscients du rôle hégémonique que leurs pays ont joué dans la région balkanique par le passé, et ont repris depuis les années 
1990 au travers d'investissements et d'implantations d'entreprises ${ }^{18}$. Si jusqu'au début du $\mathrm{XX}^{\mathrm{e}}$ siècle, la « Turquie d'Europe » désignait les Balkans, actuellement ce terme est utilisé pour les migrants turcs qui vivent en Europe. Sans l'avoir nécessairement choisi, ces derniers se trouvent ainsi associés à la domination ottomane dans les Balkans ; étant venus en Belgique dans des conditions bien moins glorieuses, ils ont trouvé dans cet héritage historique un réservoir d'images valorisantes renvoyant à une culture raffinée et à la capacité des Turcs à être du côté des grandes puissances. De ce point de vue, l'attrait des Turcs pour la référence balkanique leur permet de garder vivant ce souvenir de domination, qui les inscrit également dans l'espace européen. Or, si les Balkans constituent la preuve de l'ancrage turc dans le continent européen depuis des siècles, ils sont assimilés pour les Grecs à un modèle d'identification plutôt dévalorisant, qui les coupe du reste de l'Europe.

Grâce à leur installation à Bruxelles, les immigrés grecs et turcs (d'origine majoritairement rurale) ont pu être initiés à la culture urbaine "à l'occidentale ". Leur expérience de vie leur donne la possibilité de revendiquer tant des qualités attribuées à l'Homo Balkanicus (comme l'honneur, le fait de tenir sa parole et l'envie de préserver les traditions familiales) que des traits censés caractériser la culture occidentale (comme l'obéissance aux lois, la discipline et l'esprit d'organisation ${ }^{19}$ ). Leur mode d'auto-présentation ne met pas en cause ces stéréotypes ; au contraire, il veulent incarner la synthèse de ce qu'ils considèrent comme étant « bon à garder » de chaque côté. Quand ils ont recours à ce type de généralisations, ils n'adoptent pourtant pas une lecture monolithique : le Flamand n'est pas comme le Wallon, même si tous les deux font partie du monde occidental; de même, les Albanais sont censés être plus « durs » et moins « civilisés » que les Grecs ou les Turcs. Des hiérarchisations sont ainsi établies ${ }^{20}$, qui désignent tant la supériorité de son propre groupe ethnique que l'infériorité de ceux qui sont considérés comme incarnant une altérité à l'intérieur de chaque catégorie. Si le cadre balkanique offre la possibilité d' "être soi-même » et le contexte européen ouvre la porte à l'apprentissage des « bonnes manières », c'est la

18. Antonis Camaras (2001) a introduit l'expression de «Greek Capitalist Diaspora » afin de décrire ces nouveaux investisseurs et commerçants grecs dans les Balkans. Quant à la Turquie, elle a effectué ce qu'on a appelé « un retour dans les Balkans » depuis 1991 : «Le commerce représente précisément cet outil politique indolore qui permettrait à la Turquie de contourner les réticences d'ordre émotionnel des pays de la région » (Gangloff 2001 : 354). La crise grecque actuelle n'a pas changé la donne en ce qui concerne la relation des Grecs avec les Balkans : cette crise économique est perçue comme une affaire européenne, qui concerne principalement les pays d'Europe du sud. Ce qui change, en fait, avec la crise est la perception de l'Europe en tant qu'entité divisée entre un nord et un sud. Néanmoins, il y a de plus en plus un discours en Grèce sur les craintes de paupérisation : « ils veulent transformer la Grèce en Bulgarie où tout le monde touche des salaires de misère $»$.

19. Selon mes informateurs grecs, ces caractéristiques distinguent les migrants grecs qui ont vécu en Europe (les «Grecs d'Europe ») de leurs compatriotes qui n'ont jamais quitté le pays natal (les «Grecs de Grèce »). En ce qui concerne la crise actuelle, les Grecs de Belgique critiquent la tendance des Grecs de Grèce à ne pas payer leurs impôts, tandis que ces derniers se justifient en pointant l'inefficacité et la corruption de l'État grec.

20. Milica Bakić-Hayden (1995) a inventé le terme de «nesting Orientalisms » afin d'analyser ce jeu classificatoire. 
combinaison stratégique de ces deux alternatives qui fait la spécificité des Grecs et des Turcs vivant à Bruxelles.

Le fait de jongler entre ces deux catégories est profitable non seulement aux Grecs et aux Turcs de la diaspora, mais aussi à ceux qui habitent toujours le pays d'origine. Si le cadre balkanique constitue l'espace privilégié où leur vraie nature de « maîtres $\mathrm{du}$ jeu » peut encore se manifester, le cadre européen offre des moyens rationnels de légitimation. Surtout pour les Turcs, qui peuvent se présenter maintenant comme des agents « modernisateurs » capitalistes qui ont su rester fidèles à leurs traditions ancestrales : cela permet d'effacer l'image du « retard » que l'occupation ottomane aurait provoqué dans les Balkans, selon les différentes rhétoriques nationales dans la région. La référence balkanique offre ainsi aux Turcs la possibilité de devenir le centre de cette périphérie, au lieu de rester à la périphérie du monde occidental. Pour les Grecs qui font partie de l'Union européenne depuis 1981, le but est d'asseoir cette nouvelle forme de domination dans la région sur les deux modes d'identification : en tant qu'Européens, ils connaissent déjà la manière dont l'Union européenne fonctionne, c'est pour cela qu'ils peuvent initier leurs voisins balkaniques aux codes de ce nouvel univers ; en tant qu'hommes des Balkans, ils connaissent la manière dont les populations de cette région réagissent, et c'est pour cela qu'ils peuvent servir d'intermédiaires à leurs partenaires européens. La revendication simultanée d'un héritage balkanique et européen offre donc aux Grecs et aux Turcs un champ d'options matérielles et idéologiques : l'attachement aux Balkans leur permet d'affirmer leur pouvoir de domination, tandis que la référence à l'Europe confirme l'esprit noble et bienveillant de cette domination, en les inscrivant dans le registre de l'exercice de la suprématie occidentale.

\section{Références citées}

AleXANDris, Alexis, 1995. « La politique extérieure turque, 1923-1993 : les Balkans, le Caucase et l'Asie centrale ", in Thanos Veremis (éd.), La Turquie aujourd'hui. État, société, économie, politique extérieure, religion, 445-516. Athènes : Papazisis (en grec).

Antiochos, Anastasia, 2002-2003. L'immigration grecque dans le bassin houiller liégeois. Tentative d'une étude quantitative d'une réalité contrastée. Mémoire en Histoire contemporaine (dir. A. Morelli). Bruxelles : Université Libre de Bruxelles.

Bakić-Hayden, Milica, 1995. « Nesting Orientalisms : The Case of Former Yugoslavia », Slavic Review, 54(4) : 917-931.

Camaras, Antonis, 2001. « A Capitalist Diaspora : The Greeks in the Balkans ». Discussion paper 4. The Hellenic Observatory-LSE (electronic version).

Castellan, Georges, 2003. Un pays inconnu : La Macédoine. Hier et aujourd'hui. Crozon : Armeline.

Clayer, Nathalie, 2001. «L'islam, facteur des recompositions internes en Macédoine et au Kosovo », in Xavier Bougarel et Nathalie Clayer (éds.), Le Nouvel Islam balkanique. Les musulmans, acteurs du post-communisme. 1990-2000, 177-240. Paris : Maisonneuve et Larose.

Drezov, Kyril, 1999. « Macedonian identity : an overview of the major claims », in James Pettifer (éd.), The new Macedonian question, 47-59. London : MacMillan Press LTD.

DuiJzings, Ger, 2000. Religion and the politics of identity in Kosovo. New York : Columbia University Press. 
De Rapper, Gilles et Pierre Sintes (éds),(avec la collaboration de Kira Kaurinkoski), 2008. Nommer et classer dans les Balkans. Athènes : École française d'Athènes.

Gangloff, Sylvie, 2001. « La politique balkanique de la Turquie et le poids du passé ottoman », in Xavier Bougarel et Nathalie Clayer (éds.), Le nouvel Islam balkanique. Les musulmans, acteurs du postcommunisme. 1990-2000,317-356. Paris : Maisonneuve et Larose.

Garde, Paul, 2004. Le discours balkanique. Des mots et des hommes. Paris : Fayard.

Gossiaux, Jean-François, 2002. Pouvoirs ethniques dans les Balkans. Paris : Presses Universitaires de France.

Gravier, Gaston, 1913. «L'Albanie et ses limites », Revue de Paris, 1 et 15 janvier : 200-224 et $443-448$.

Karakassidou, Anastasia N., 2004. «Affections of a Greek hero. Pavlos Melas and heroic representations in Greece », in Maria Todorova (éd.), Balkan identities. Nation and memory, 197-232. London : Hurst and Company.

KisACIK, Fendiye, 2000-2001. L'immigration turque dans l'industrie charbonnière belge. Éléments pour la compréhension de la politique de recrutement et de gestion de la main-d'œuvre, Mémoire en Histoire contemporaine (dir. A. Morelli), Bruxelles, Université Libre de Bruxelles.

Mandel, Ruth, 2008. Cosmopolitan anxieties. Turkish challenges to citizenship and belonging in Germany. Durham - Londres : Duke University Press.

Martens, Albert, 1976. Les immigrés. Flux et reflux d'une main-d'oeuvre d'appoint. La politique belge de l'immigration de 1945 à 1970. Louvain : Presses universitaires de Louvain.

Moutsou, Christina, 1998. Handling «food for thought» : Greek and Turkish hybrid representations in Brussels. PhD dissertation, University of Cambridge.

Parthoens, Christophe et Altay Manço, 2005. De Zola à Atatürk : un « village musulman » en Wallonie. Paris : L'Harmattan.

Petridou, Elia, 2005. " Au pays de la feta. Négociation de la grécité dans le contexte européen », Ethnologie française, XXXV (2) : 255-265.

Prevelakis, Georges, 2002. « Hellénisation des Balkans ou balkanisation de la Grèce ? », in Stéphane Yérasimos (éd.), Le retour des Balkans. 1991-2001, 131-143. Paris : Autrement.

Roux, Michel, 1992. Les Albanais en Yougoslavie. Minorité nationale, territoire et développement. Paris : Éditions de la Maison des Sciences de l'Homme.

Seraidari, Katerina, 2011. «Transactions commerciales et relations sociales entre Grecs et Turcs de Bruxelles », Brussels Studies, 53 : 1-12.

Taschereau, Sylvie, Valérie Piette et Eliane Gubin, 2001. « L'immigration à Bruxelles dans les années trente. Le cas particulier des commerçants étrangers », Cahiers d'Histoire du temps présent, 9 : $7-62$. 